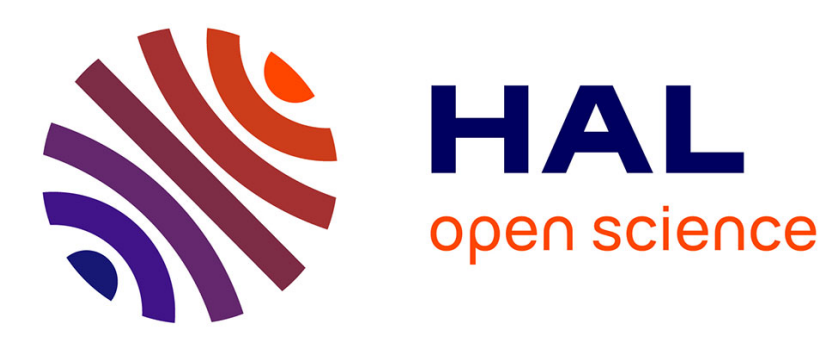

\title{
Scalp EEG Activity During Simple and Combined Motor Imageries to Control a Robotic Arm
}

Cecilia Lindig-Leon, Sébastien Rimbert, Oleksii Avilov, Laurent Bougrain

\section{To cite this version:}

Cecilia Lindig-Leon, Sébastien Rimbert, Oleksii Avilov, Laurent Bougrain. Scalp EEG Activity During Simple and Combined Motor Imageries to Control a Robotic Arm. 2017 IEEE First Ukraine Conference on Electrical and Computer Engineering, May 2017, Kiev, Ukraine. hal-01519712

\section{HAL Id: hal-01519712 \\ https://hal.inria.fr/hal-01519712}

Submitted on 9 May 2017

HAL is a multi-disciplinary open access archive for the deposit and dissemination of scientific research documents, whether they are published or not. The documents may come from teaching and research institutions in France or abroad, or from public or private research centers.
L'archive ouverte pluridisciplinaire HAL, est destinée au dépôt et à la diffusion de documents scientifiques de niveau recherche, publiés ou non, émanant des établissements d'enseignement et de recherche français ou étrangers, des laboratoires publics ou privés. 


\title{
Scalp EEG Activity During Simple and Combined Motor Imageries to Control a Robotic Arm
}

\author{
Cecilia Lindig-Leon ${ }^{1,2,3}$, Sébastien Rimbert ${ }^{1,2,3}$, Oleksii Avilov ${ }^{4,1,2,3}$, and Laurent Bougrain ${ }^{2,1,3}$ \\ ${ }^{1}$ Neurosys team, Inria, Villers-lès-Nancy, F-54600, France \\ ${ }^{2}$ Artificial Intelligence and Complex Systems, Université de Lorraine, LORIA, UMR 7503, Vandœuvre-lès-Nancy, F-54506, France \\ ${ }^{3}$ Neurosys team CNRS, LORIA, UMR 7503, Vandœuvre-lès-Nancy, F-54506, France \\ ${ }^{4}$ Electronic Dept., National Technical University of Ukraine "Igor Sikorsky Kyiv Polytechnic Institute", Kyiv, Ukraine \\ Email: cecilia.lindig-leon@inria.fr, sebastien.rimbert@inria.fr, lesha.avilov@gmail.com, laurent.bougrain@loria.fr
}

\begin{abstract}
In this article, we study how combined motor imageries can be detected to deliver more commands in a Brain-Computer Interface for controlling a robotic arm. Motor imageries are a major way to deliver commands in BCI. Nevertheless only a few systems use more than three motor imageries: right hand, left hand and feet. Combining them allow to get four additional commands. We present an electrophysiological study to show that i) simple motor imageries have mainly an electrical modulation over the cortical area related the body part involved in the imagined movement and that ii) combined motor imageries reflect a superposition of the electrical activity of simple motor imageries. A shrinkage linear discriminant analysis has been used to test as a first step how a resting state and seven motor imageries can be detected. 11 healthy subjects participated in the experiment for which an intuitive assignment has been done to associate motor imageries and movements of the robotic arm with 7 degrees of freedom.
\end{abstract}

\section{INTRODUCTION}

Motor imagery (MI) is the ability to imagine performing a movement without executing it [1]. According to Jeannerod [2], MI represents the result of conscious access to the content of the intention of a movement, which is usually performed unconsciously during movement preparation [3]. MI has two different components, namely the visual-motor imagery and the kinesthetic motor imagery (KMI) [4]. KMI generates an event-related desynchronization (ERD) and an event-related synchronization (ERS) in the contralateral sensorimotor area, which is similar to the one observed during the preparation of a real movement (RM) [5]. More precisely, compared to a resting state taken before a motor imagery, several power modulations are observed in in the alpha (7$13 \mathrm{~Hz})$ and in the beta $(18-25 \mathrm{~Hz})$ bands of the electroencephalographic signal measured over the sensorimotor area corresponding to the body part involved in the motor imagery. Firstly there is a gradual power decrease in the alpha and in the beta bands, called ERD. Secondly, a low power level is maintained during the movement. Finally, from 300 to 500 milliseconds after the end of the motor imagery, there is a power increase called ERS or post-movement beta rebound with a duration of about one second. Although several studies showed an activity uniquely in the contralateral area [6], other studies showed that ERD and ERS are also in the ipsilateral area [7].
Emergence of ERD and ERS patterns during and after a MI has been intensively studied in the Brain-Computer Interface (BCI) domain [8] in order to define detectable commands for the system. BCI systems consist of a combination of modules that allow recording the user brain activity in order to analyze it and find specific patterns indicating that the person is executing a particular mental task, which in turn is associated to a specific command for controlling the function of certain devices, such as computers, robotics, wheelchairs, prostheses, orthoses, etc. In this way, BCIs replace the natural nervous system pathways by artificial ones, which allow people with a severe motor impairment to interact with their environment by using only their brain activity. Most of BCI applications aim at improving the quality of life of their users, who have lost certain function(s) due to a degenerative disease or an accident, in which case the system will attempt to restore the missing ability.

In order to explore new solutions for overcoming the existing limitations of EEG-based neuroprostheses, we explored new alternative that have been implemented and motivated by a JACO 3-fingers robotic arm. This study consists to control the three body parts of this robotic arm in increasing the number of MI commands. Indeed, we will present a 8class database; which comprises EEG signals from 11 healthy subjects inducing the eight mental states that it is possible to generate by the motor tasks involving the use of the right hand, left hand, both feet together, and rest. We will present a qualitative analysis on the ERD patterns that are found in the EEG signals during the eight different tasks and the classification performance for each subjects.

\section{Material AND Methods}

\section{A. The JACO 3-fingers robotic arm}

The proposed approach has been motivated by a commercial device model JACO 3 -fingers by Kinova ${ }^{\mathrm{TM} 1}$, which affords a full 3D control through fourteen predefined motions; including six reaching movements along the three axis, six rotations around the three axis, and a grasping function. These functions are distributed in three main articulations-like that resemble the

\footnotetext{
${ }^{1}$ http://www.kinovarobotics.com/assistive-robotics/products/manipulation/
} 
forearm, with which the device is able of approaching objects, the wrist, with which it is possible to turn the hand towards any direction, and the fingers, which allows to open and close the hand (Figure 1). Regarding the use of the fingers, even when the device allows controlling each pair separately, we have decided to consider them all together. In this way the number of required commands decreases without loss of functionality, since the grasping action by using two or three of the fingers has almost the same effect.

A)

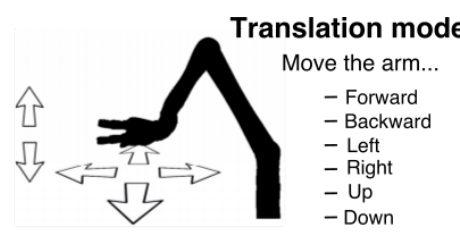

Wrist mode

Movement of the wrist..

- Orientation top

- Orientation bottom

- Thumb side orientation

- Index side orientation

- Rotation of the wrist (left)

- Rotation of the wrist (right)

Finger mode

Grasping movement...

- Open three fingers

C)

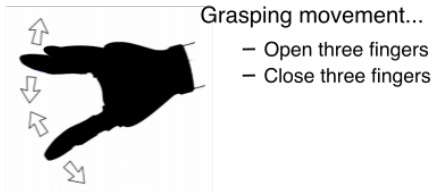

Fig. 1. 3-axis operation mode. The robotic arm affords a full 3D control through fourteen predefined motions; including six reaching movements along the three axis (A), six rotations around the three axis (B), and a grasping function (C). These functions are distributed in three main articulations-like that resemble the forearm, the wrist, and the fingers. Note that we have decided to use all fingers together, so that the number of required commands decreases without loss of functionality, since the grasping action by using two or three of the fingers has almost the same effect.

1) Implementation challenges: The appropriate use of this robotic arm implies several challenges that must be addressed from different perspectives. For instance, users' training is one of the most difficult aspects. Even when powerful feature extraction and classification techniques are implemented, the control of this kind of devices becomes unsatisfactory if users are not able of properly modulating their brain activity. In this sense, the proposed paradigm adds a greater difficulty in comparison to the standard protocols, since the movement imagination of more than one body part at the same time requires a higher degree of coordination. Furthermore, training sessions can be long and tedious for users, specially if the task is not well accomplished, which might cause frustration and make the training process even more difficult. It must be mentioned that this aspect represents one of the main areas of BCI research, which was beyond of the scope of the present project and, therefore, it remains to be addressed as part of the related feature work.

2) Multiple commands: As shown in Figure 1 this device offers several predefined motions, which on the one hand facilitates the interaction with the subject, but on the other hand demands a considerable number of different brain states to be properly induced and classified. In this regard there are at least two aspects to be considered; first, the number of body parts with which it is possible to offer an intuitive control and whose related sources are separated enough to distinguish the induced activity is limited, and second, the classification task becomes more difficult as the amount of classes increases, which can lead to misclassified responses that hinder the correct use of the device. Because of these considerations we have decided to explore the use of combined motor imageries, since in contrast with the standard scheme, this approach has the advantage of considerably increasing the amount of afforded commands while using the same number of body parts (in order of $2^{P}$ compared to $P$, where $P$ is the number of body parts). However, even when the use of combined movements is considered, there might be not enough commands to control devices as the one we are describing. For instance, in the present work we have decided to consider the use of the left hand, right hand and both feet together. Given that these body parts are intuitively associated with movement. It is worth mentioning that feet are considered together given the proximity of the two sources, which may be difficult to distinguish.

Under this scheme users are afforded only with eight commands if the rest condition is also considered (i.e., left hand, feet, left hand in combination with feet, right hand, both hands together; right hand in combination with feet; both hands together with feet, and rest), which are not sufficient to control the fourteen available motions. To overcome the insufficiency of commands we have designed a smart switching-mode scheme that allows controlling different actions by using the same command.

The combination of the three body parts included in the paradigm together with the rest condition, afford users with eight different commands. However, since it is essential to keep the rest condition unassigned to any movement, only seven classes remain to control the device. To this end, an implementation based on three modes has been proposed: one mode for controlling the arm position (see Figure 1-A), another one for the wrist (see Figure 1-B), and the third one for opening and closing the fingers (see Figure 1-C). Each mode assigns a specific class to a specific motion. The combination of the three motor imageries (both hands and feet) has been established to be used as a switch that allows changing from one mode to the next one (arm, wrist, fingers, arm...). In this way it is possible to manage different actions by using the same mental command to control the fourteen available motions (see Figure 2).

\section{B. Participants}

Eleven right-handed healthy volunteer subjects took part in this experiment ( 8 men and 3 women, 19 to 43 years old). They had no medical history which could have influenced the task. All experiments were carried out with the consent agreement (approved by the ethical committee of INRIA) of each partici- 


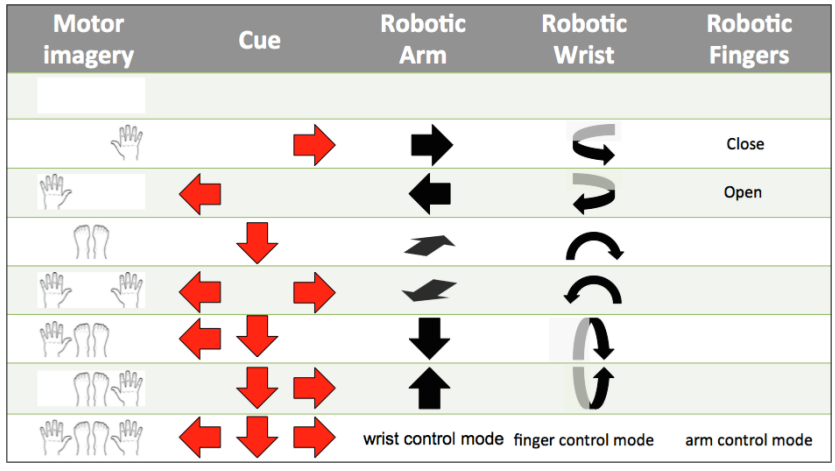

Fig. 2. Arm control. The different MIs that users were asked to perform are shown in the first column with their associated cue stimuli in the second one The last three columns present the different robotic movements associated to each mode. Note that the rest condition is not linked to any action, and that motor imagery including the combination of all body parts represents a switch to change from one mode to another.

pant and following the statements of the WMA declaration of Helsinki on ethical principles for medical research involving human subjects [9].

\section{Protocol}

Subjects were seated in a comfortable chair with the arms at their sides in front of a screen showing the task cue to be performed, which consisted of one of the eight kinesthetics motor imageries that it is possible to generate with all combinations including the use of the right hand, left hand, both feet together, and rest (i.e., right hand; left hand; feet; both hands together; left hand in combination with feet; right hand in combination with feet; both hands together with feet, and rest). The whole session consisted of 4 runs, containing each one 10 trials per task, which totals 40 trials per class (320 trials considering the 8 classes). For the stimulation, three panels were simultaneously displayed on the screen, which were associated from left to right, to the left hand, feet and right hand. Each trial was randomly presented and lasted for 12 seconds, starting at second 0 with a cross at the center of each panel and an overlaid arrow indicating for the next 6 seconds the task to be performed: an arrow pointing to the left side on the left panel for left hand, an arrow pointing down on the central panel for feet, an arrow pointing to the right side on the right panel for right hand, and the simultaneous combination of all of them for the corresponding combined tasks (see Figure 3 and column 2 of Figure 2). The rest condition was also considered and it was indicated by the absence of arrows. After second 6, the task cue disappeared and the crosses were remaining for the next 6 seconds indicating the pause period before the next trial started.

\section{Electrophysiological data}

EEG signals were recorded by the OpenViBE [10] platform from fiftenn right-handed healthy subjects at $256 \mathrm{~Hz}$ using a commercial REFA amplifier developed by TMS International $^{\mathrm{TM}}$. The EEG cap was fitted with 26 passive electrodes, namely $\mathrm{Fp} 1 ; \mathrm{Fp}_{\mathrm{z}} ; \mathrm{Fp} 2 ; \mathrm{F}_{\mathrm{z}} ; \mathrm{FC} 5 ; \mathrm{FC} 3 ; \mathrm{FC} 1 ; \mathrm{FC}_{\mathrm{z}}$;

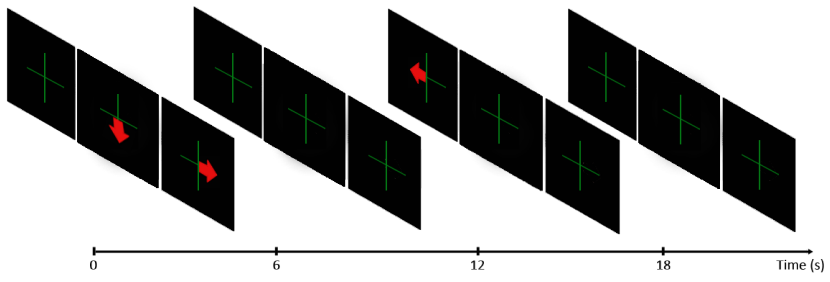

Fig. 3. Time scheme. Each trial was randomly presented and lasted for 12 seconds. During the first 6 seconds, users were asked to perform the motor imagery indicated by the task cue, which was launched throughout three simultaneously displayed panels associated, from left to right, to left hand, feet and right hand. The use of each body part was indicated by the presence of arrows: an arrow pointing to the left side on the left panel for left hand, an arrow pointing down on the central panel for feet, an arrow pointing to the right side on the right panel for right hand, and the simultaneous combination of all of them for the corresponding compound motor imageries. After second 6 , the task cue disappeared and the crosses were remaining for the next 6 seconds indicating the pause period before the next trial started.

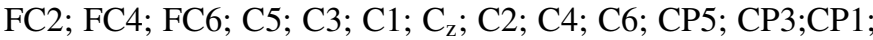
$\mathrm{CP}_{\mathrm{z}} ; \mathrm{CP} 2 ; \mathrm{CP} 4 ; \mathrm{CP} 6$ and $\mathrm{P}_{\mathrm{z}}$, re-referenced with respect to the common average reference across all channels and placed by using the international 10-20 system positions to cover the primary sensorimotor cortex.

\section{E. ERD/ERS patterns}

To evaluate more precisely the modulation which appeared during the two different time windows, we computed the ERD/ERS\% using the "band power method" [5] with a Matlab code. First, the EEG signal was filtered in the mu frequency band $(7-13 \mathrm{~Hz})$ for all subjects using a 4th-order Butterworth band-pass filter. Then, the signal was squared for each trial and averaged over trials. Then it is smoothed using a 250ms sliding window with a $125 \mathrm{~ms}$ shifting step. The averaged power computed for each window was subtracted and then divided by the averaged power of a baseline corresponding to a $2 s$ window before each trial. Finally, the averaged power computed for each window was subtracted and then divided by the averaged power of a baseline corresponding $2 \mathrm{~s}$ before each trial. Trials were considered 2 seconds before the task cue appeared on the screen and 6 seconds after it disappeared, so that each segment corresponds to a 14s-length signal segment over 26 electrodes (Figure 5, Figure 6 and Figure 7).

\section{F. Classification}

Each subject performed 4 runs of motor imageries. Each run contains 10 trials for each class (Rest, Left Hand, Right Hand, Both hands, Feet, Left hand \& Feet, Right hand \& Feet, Both hands \& Feet). Thus 40 trials per class are available for each subject. From each trial 26 features (one per channel) have been extracted from the filtered signal computing the logarithm of the variance of the amplitude values between 0.5 and 3.5 s after the GO signal. For classification a 8-classes Shrinkage Linear Discriminant Analysis (sLDA) [11] has been used via the python library scikit-learn [12]. The reason to use a shrinkage estimator is due to the small number of samples per class. For the same reason, a cross validation has been 
done using 3 runs for train and 1 run for test. Thus 240 trials for train and 80 trials for test for every step of a the cross validation.

\section{RESULTS}

\section{A. Oscillatory power analysis}

Figure 4 shows a topographic representation for one of the subjects along all conditions of the ERD/ERS\% values obtained for all electrodes and averaged over the 4-second period starting one second after the task cue was presented. It can be observed that the lowest values appear on the regions associated to the limbs that are engaged at each motor task (broadly, right hand over electrode $\mathrm{C} 3$, feet over electrode $\mathrm{C}_{\mathrm{z}}$, and left hand over electrode $\mathrm{C} 4$ ).

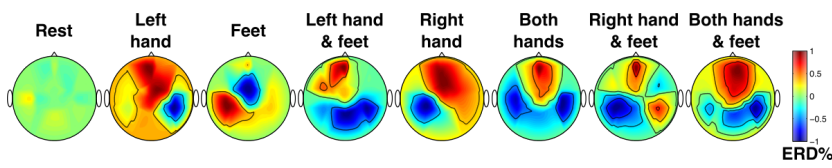

Fig. 4. Distribution of the relative oscillatory power along the sensorimotor cortex during the course of the different motor tasks for subjet 2. ERD/ERS\% values were obtained for all electrodes within the mu frequency range and averaged over the 4-second period starting one second after the task cue was presented. Negative values indicate ERD\% modulation, which is characteristic of a motor task execution that appears over the contralateral hemisphere of the body part used in the process (broadly, electrode $\mathrm{C} 4$ for left hand, $\mathrm{C}_{\mathrm{z}}$ for feet, and C3 for right hand). Note that each topographic map is independently normalized to enhance the corresponding patterns.

If we take a closer look over the time course of ERD/ERS\% over electrode C3 (i.e, the activity source associated with the use of the right hand) presented in Figure 5, we can observe that all motor imageries involving the use of the right hand (i.e., right hand, right hand in combination with feet, both hands, and both hands in combination with feet) present considerably lower ERD/ERS\% values than those that do not include it (i.e., rest, left hand, feet, and left hand in combination with feet).

Similarly, Figure 6 presents the time course of ERD/ERS\% over electrode $C_{z}$ (i.e, the activity source associated with the use of the feet). Over this region it can also be observed that the motor imageries involving the use of the feet (i.e., feet, left hand in combination with feet, right hand in combination with feet, and both hands in combination with feet) present lower ERD/ERS\% values than those that do no it include it (i.e., rest, left hand, right hand, and both hands). However, in comparison to the contrast observed over electrode $\mathrm{C} 3$ between the tasks involving the use of the right hand and those that do not involve it, differences are not as significant. In fact, we can observe that the use of both left and right hands induce some desynchronization over the central region, possible because the associated sources are quite close to electrode $\mathrm{C}_{\mathrm{z}}$, which might cause it to incorporate some of this activity.

Finally, the same comparison is illustrated in Figure 7 for the time course of ERD/ERS\% generated over electrode C4 (i.e, the activity source linked to the use of the left hand). In this case, similarly to the analysis over electrode $\mathrm{C} 3$, it is possible to observe a significant contrast between the low
ERD/ERS\% values obtained from the activity induced by the motor imageries involving the use of the left hand (i.e., left hand, left hand in combination with feet, both hands, and both hands in combination with feet) from the high ERD/ERS\% values associated to the tasks that do not involve the use of the left hand (i.e., rest, feet, right hand, and right hand in combination with feet).

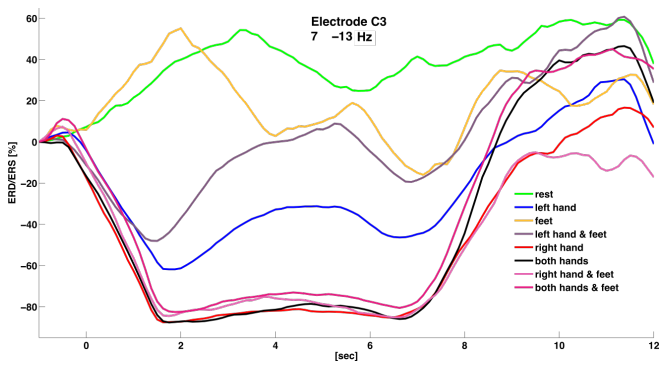

Fig. 5. ERD/ERS\% time series within the mu range for all motor tasks over electrode $\mathrm{C} 3$ (i.e, the activity source associated with the use of the right hand). ERD/ERS values were estimated over the EEG trials from subject 2. Note that all motor imageries involving the use of the right hand (i.e., right hand, right hand in combination with feet, both hands, and both hands in combination with feet) present considerably lower values that those that do not include it (i.e., rest, left hand, feet, and left hand in combination with feet)

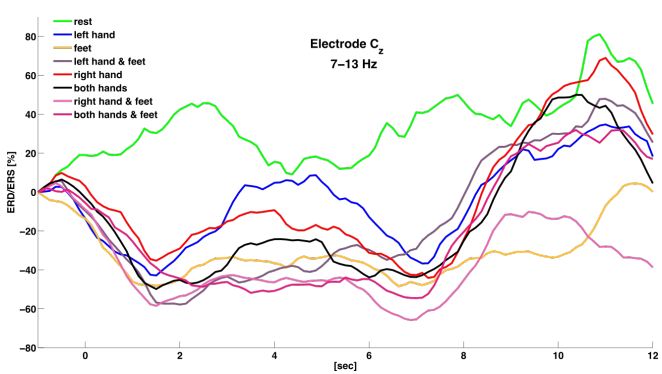

Fig. 6. ERD/ERS\% time series within the mu range for all motor tasks over electrode $C_{z}$. ERD/ERS values were estimated over the EEG trials from subject 2. Note that the motor imageries involving the use of the feet (i.e., feet, left hand in combination with feet, right hand in combination with feet, and both hands in combination with feet) present lower ERD/ERS\% values than those that do no it include it (i.e., rest, left hand, right hand, and both hands). However, this contrast is not as significant as the one observed on electrode $\mathrm{C} 3$, possibly due to the fact that the regions associated to both the left and right hands are quite close to electrode $C_{z}$, which might cause it to incorporate some of this activity.

\section{B. Statistical analysis}

We present a series of box plots for comparing the power spectrum magnitudes at $12 \mathrm{~Hz}$ between all motor tasks over the regions associated to the main sources. Again, it is possible to observe that motor imageries involving the use of the limb associated to each electrode present similar low power values at these positions, whether those that are not engaged generate higher values. This is particularly visible from figure 8 , which presents the corresponding analysis over electrode C3 (i.e., the region associated to the right hand). Note that the distribution of the mean values confirm that there is no significant difference between motor imageries including the use of the right hand over this region, independently from 


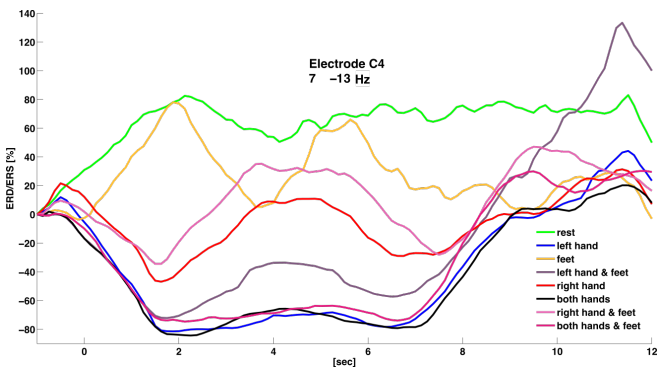

Fig. 7. ERD/ERS\% time series within the mu range for all motor tasks over electrode C4. ERD/ERS values were estimated over the EEG trials from subject 2. Note that all motor imageries involving the use of the left hand (i.e., left hand, left hand in combination with feet, both hands, and both hands in combination with feet) present considerably lower values that those that do not include it (i.e., rest, right hand, feet, and right hand in combination with feet).

the fact of whether the motor imagery is simple or combined. In contrast there is a significant difference with all the other tasks that do not include the use of the right hand which, in turn, present a uniform distribution between them. The same analysis is presented for electrode $C_{z}$ (i.e., the region associated to the feet) and electrode $\mathrm{C} 4$ (i.e., the region associated to the left hand) in figures 9 and 10 respectively.

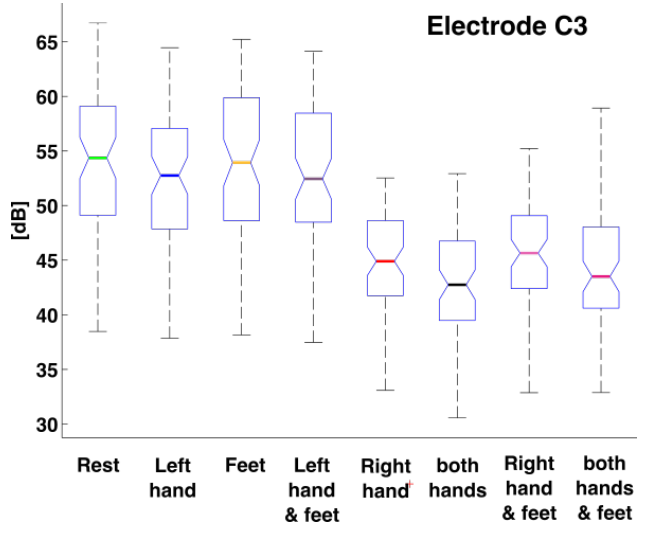

Fig. 8. Box plots for subjet 2 comparing the power spectrum magnitude at 12 $\mathrm{Hz}$ within the time interval from [2-5 s] among all the considered conditions over electrode $\mathrm{C} 3$. The distribution of data shows that there is no significant difference between motor imageries including the use of the right hand over this region, independently from the fact of whether the motor imagery is simple or combined. In contrast there is a significant difference with all the other tasks that do not include the use of the right hand which, in turn, present a uniform distribution between them.

\section{Classification}

Table I reports the accuracy for 8 classes obtained by a shrinkage LDA for 11 subjects. The mean accuracy of 31.6 $\%$ is low even compared to a random assignment (12.5\%). However, it should be noted that two subjects have better performance (S2 and S11) compared with other subjects. It is interesting to see that the best subject in term of accuracy had also the powerful ERD during the MI-task (Figure 5, Figure 6 and Figure 7). Thus, in the next section, we will discuss possible solutions to improve the classification accuracy.

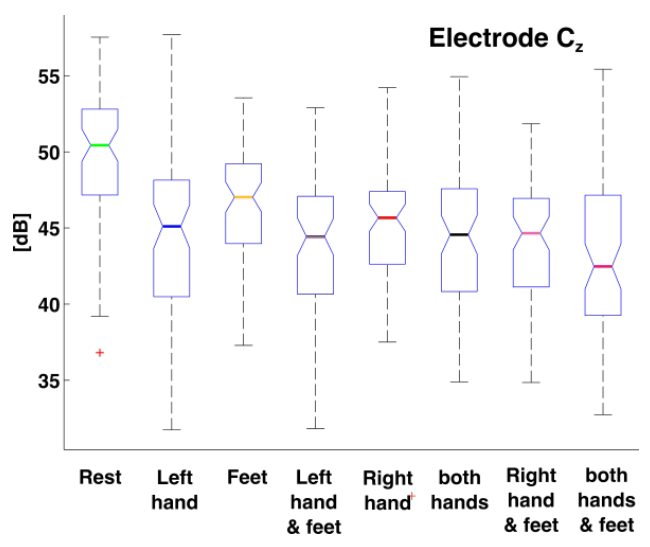

Fig. 9. Box plots for subjet 2 comparing the power spectrum magnitude at 12 $\mathrm{Hz}$ within the time interval from [2-5 s] among all the considered conditions over electrode $\mathrm{C}_{\mathrm{z}}$. The distribution of data reflects what was observed from the ERD/ERS\% time series, and confirms that EEG activity around the central region is influenced by the surrounding sources.

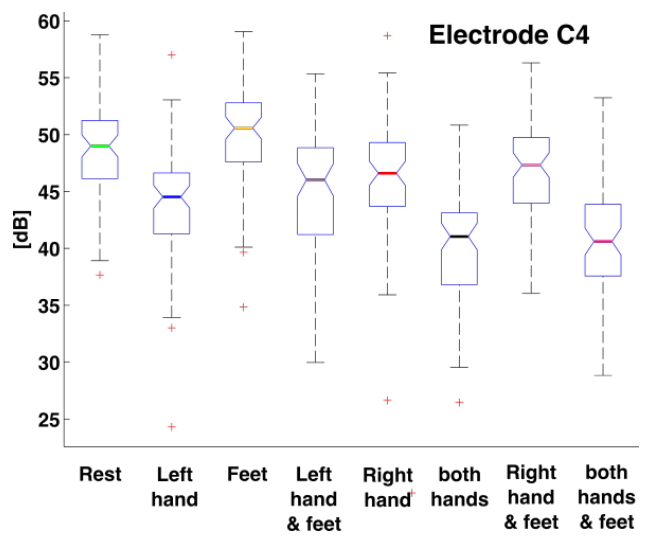

Fig. 10. Box plots for subjet 2 comparing the power spectrum magnitude at 12 $\mathrm{Hz}$ within the time interval from [2-5 s] among all the considered conditions over electrode $\mathrm{C} 4$. The distribution of data shows that there is no significant difference between motor imageries including the use of the left hand over this region, independently from the fact of whether the motor imagery is simple or combined. In contrast there is a significant difference with all the other tasks that do not include the use of the left hand which, in turn, present a uniform distribution between them.

Table II shows the main misclassifications. In particular, feet motor imageries have been confused with a resting state (Rest $\rightarrow$ Feet, Feet $\rightarrow$ Rest, Both hands $\rightarrow$ Feet, Left hand \& Feet $\rightarrow$ Left hand, Right hand \& Feet $\rightarrow$ Right hand, Both hands \& Feet $\rightarrow$ Both hand). One explanation could be that the activity of the right hand (respectively the left hand and both hands) goes over the $\mathrm{Cz}$ location.

Each class is described by a few number of samples (30 for training and 10 for testing). Increase the number of samples for each class should improve the performance. The low classification rate could also be linked with the difficulty to properly imagine a kinesthetic movement. An incremental motor imagery learning using first real movements and then intented movements could be useful to train the user to produce a good motor imagery. 
TABLE I

ACCURACY OBTAINED BY A SHRINKAGE LDA FOR EACH SUBJECT FOR 8-CLASS OF MOTOR IMAGERIES.

\begin{tabular}{|l|l|}
\hline Subject & 8-class accuracy \\
\hline 1 & $21.9 \% \pm 3.4 \%$ \\
\hline 2 & $58.1 \% \pm 5 \%$ \\
\hline 3 & $34.7 \% \pm 2.7 \%$ \\
\hline 4 & $28.4 \% \pm 6.6 \%$ \\
\hline 5 & $26.9 \% \pm 4 \%$ \\
\hline 6 & $23.7 \% \pm 1.8 \%$ \\
\hline 7 & $27.8 \% \pm 6.6 \%$ \\
\hline 8 & $26.6 \% \pm 3.5 \%$ \\
\hline 9 & $20.3 \% \pm 2 \%$ \\
\hline 10 & $35.3 \% \pm 3 \%$ \\
\hline 11 & $43.4 \% \pm 5.2 \%$ \\
\hline AVG & $\mathbf{3 1 . 6 \%} \pm \mathbf{1 0 . 5 \%}$ \\
\hline
\end{tabular}

\section{CONCLUSION}

To control a robotic arm with 14 commands using a EEGbased BCI, we presented a mapping of these commands with a set of simple and combined motor imageries. Our study shows that the electrical activity of combined motor imageries measured on the scalp is very similar to the electrical activity measured for each simple motor imagery taken individually. Thus an efficient classification should be able to distinguish them. The accuracy obtained by a shrinkage linear discriminant analysis is not good enough to allow a control of the robotic arm. Further works will focus on ensemble of binary classifiers to allow the use the efficient common spatial pattern algorithm [13] as pre-processing to increase by linear combination of the channels the amplitude of the signal for one class and decrease it for the other one. Then one-versusone approach, one-versus-the-rest approach or a hierarchical approach should improve the detection of combined motor imageries.

\section{REFERENCES}

[1] L. Avanzino, A. Giannini, A. Tacchino, E. Pelosin, P. Ruggeri, and M. Bove, "Motor imagery influences the execution of repetitive finger opposition movements," Neuroscience Letters, vol. 466, no. 1, pp. $11-$ $15,2009$.

[2] M. Jeannerod, "Mental imagery in the motor context," Neuropsychologia, vol. 33, no. 11, pp. 1419-32, Nov 1995.

[3] M. Lotze and U. Halsband, "Motor imagery," J Physiol Paris, vol. 99, no. 4-6, pp. 386-95, Jun 2006.
[4] C. Neuper, R. Scherer, M. Reiner, and G. Pfurtscheller, "Imagery of motor actions: Differential effects of kinesthetic and visualmotor mode of imagery in single-trial EEG," Cognitive Brain Research, vol. 25, no. 3, pp. 668 - 677, 2005. [Online]. Available: http://www.sciencedirect.com/science/article/pii/S0926641005002533

[5] G. Pfurtscheller and F. H. Lopes da Silva, "Event-related EEG/MEG synchronization and desynchronization: basic principles," Clin Neurophysiol, vol. 110, no. 11, pp. 1842-57, Nov 1999.

[6] G. Pfurtscheller and C. Neuper, "Motor imagery and direct braincomputer communication," Proceedings of the IEEE, vol. 89, no. 7, pp. 1123 -1134, Jul. 2001.

[7] S. Fok, R. Schwartz, M. Wronkiewicz, C. Holmes, J. Zhang, T. Somers, D. Bundy, and E. Leuthardt, "An eeg-based brain computer interface for rehabilitation and restoration of hand control following stroke using ipsilateral cortical physiology." Conf Proc IEEE Eng Med Biol Soc, vol. 2011, pp. 6277-6280, 2011.

[8] J. Wolpaw and E. W. Wolpaw, Eds., Brain-Computer Interfaces: Principles and Practice. Oxford university press, 2012.

[9] . World Medical Association, "World medical association declaration of Helsinki: ethical principles for medical research involving human subjects." J Postgrad Med, vol. 48, no. 3, pp. 206-208, Jul-Sep 2002, kIE: KIE Bib: human experimentation.

[10] Y. Renard, F. Lotte, G. Gibert, M. Congedo, E. Maby, V. Delannoy, O. Bertrand, and A. Lécuyer, "Openvibe: An open-source software platform to design, test and use brain-computer interfaces in real and virtual environments," Presence : teleoperators and virtual environments, vol. 10, no. 1, 2010.

[11] J. H. Friedman, "Regularized Discriminant Analysis," Journal of the American Statistical Association, vol. 84, no. 405, pp. 165-175, 1989. [Online]. Available: http://dx.doi.org/10.2307/2289860

[12] F. Pedregosa, G. Varoquaux, A. Gramfort, V. Michel, B. Thirion, O. Grisel, M. Blondel, P. Prettenhofer, R. Weiss, V. Dubourg, J. Vanderplas, A. Passos, D. Cournapeau, M. Brucher, M. Perrot, and E. Duchesnay, "Scikit-learn: Machine learning in Python," Journal of Machine Learning Research, vol. 12, pp. 2825-2830, 2011.

[13] B. Blankertz, R. Tomioka, S. Lemm, M. Kawanaba, and K. Müller, "Optimizing spatial filters for robust EEG single-trial analysis [revealing tricks of the trade]," IEEE Signal processing magazine, 2008.

TABLE II

CONFUSION MATRIX (\%) OVER ALL SUBJECTS

\begin{tabular}{|c|c|c|c|c|c|c|c|c|c|}
\hline & \multicolumn{8}{|c|}{ Predicted labels } \\
\hline & & Rest & Left hand & Right hand & Both hands & Feet & Left hand \& Feet & Right hand \& Feet & Both hands \& Feet \\
\hline \multirow[b]{8}{*}{ True labels } & Rest & 44 & 5 & 7 & 5 & 18 & 9 & 6 & 6 \\
\hline & Left hand & 6 & 22 & 11 & 15 & 7 & 16 & 9 & 14 \\
\hline & Right hand & 6 & 10 & 25 & 17 & 9 & 6 & 16 & 11 \\
\hline & Both hands & 5 & 11 & 12 & 28 & 7 & 10 & 8 & 19 \\
\hline & Feet & 17 & 6 & 8 & 5 & 42 & 8 & 9 & 5 \\
\hline & Left hand \& Feet & 6 & 13 & 6 & 9 & 11 & 35 & 9 & 11 \\
\hline & Right hand \& Feet & 6 & 8 & 14 & 9 & 7 & 9 & 36 & 11 \\
\hline & Both hands \& Feet & 6 & 13 & 9 & 20 & 6 & 12 & 11 & 23 \\
\hline
\end{tabular}

